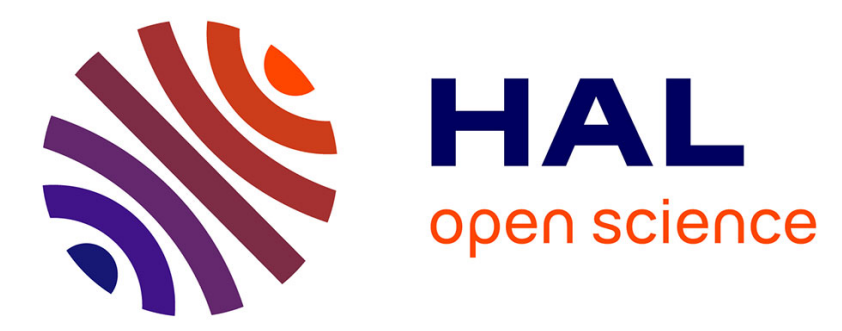

\title{
Simultaneous information and power transfer in wireless cooperative networks
}

\author{
Zhiguo Ding, Samir M. Perlaza, Iñaki Esnaola, H. Vincent Poor
}

\section{To cite this version:}

Zhiguo Ding, Samir M. Perlaza, Iñaki Esnaola, H. Vincent Poor. Simultaneous information and power transfer in wireless cooperative networks. 8th International ICST Conference on Communications and Networking in China (CHINACOM), Aug 2013, Guilin, China. 10.1109/ChinaCom.2013.6694600 . hal-01281031

\section{HAL Id: hal-01281031 \\ https://hal.science/hal-01281031}

Submitted on 1 Mar 2016

HAL is a multi-disciplinary open access archive for the deposit and dissemination of scientific research documents, whether they are published or not. The documents may come from teaching and research institutions in France or abroad, or from public or private research centers.
L'archive ouverte pluridisciplinaire HAL, est destinée au dépôt et à la diffusion de documents scientifiques de niveau recherche, publiés ou non, émanant des établissements d'enseignement et de recherche français ou étrangers, des laboratoires publics ou privés. 


\title{
Simultaneous Information and Power Transfer in Wireless Cooperative Networks
}

\author{
Zhiguo Ding ${ }^{1,2}$, Samir M. Perlaza ${ }^{2}$, Iñaki Esnaola ${ }^{2}$ and H. Vincent Poor $^{2}$ \\ ${ }^{1}$ School of Electrical, Electronic, and Computer Engineering Newcastle University, NE1 7RU, UK \\ ${ }^{2}$ Department of Electrical Engineering, Princeton University, Princeton, NJ 08544, USA. \\ (invited paper)
}

\begin{abstract}
Simultaneous power and signal transfer over wireless channels is particularly important to communication scenarios with constrained energy, such as wireless sensor networks. This paper considers a wireless cooperative network with multiple pairs of sources and destinations communicating via a relay. The impact of various strategies of power allocation on the end-to-end transmission reliability in this cooperative network is studied. Both analytical and numerical results are developed to facilitate the comparison among the different power allocation strategies.
\end{abstract}

\section{INTRODUCTION}

Recently a new concept of energy harvesting based on collecting energy from ambient radio frequency signals has been proposed [1], [2]. More specifically wireless signals can be used as a means for the delivery of information and power simultaneously. In addition, such an approach can also reduce the cost of communication networks, since peripheral equipment to take advantage of external energy sources can be avoided. The concept of simultaneous power and information delivery was first proposed in [1], where the fundamental tradeoff between the energy and information rate is characterized for point-to-point communication scenarios. The extension of such a concept to frequency selective channels is considered in [2]. In [3] the authors study energy harvesting for communication scenarios with co-channel interference, where such interference is identified as a potential energy source. The simultaneous transfer of power and information is also studied in multiple-input multiple-output systems in [4], and its extension to the scenario with imperfect channel information at the transmitter was considered in [5].

This paper considers a general wireless cooperative network, in which multiple pairs of sources and destinations communicate through an energy harvesting relay. Specifically, multiple sources deliver their information to the relay via orthogonal channels, such as different time slots. The relaying transmissions are powered by the signals sent from the sources. Assuming that the battery of the relay is sufficiently large, the relay can accumulate a large amount of power for relaying transmissions. The aim of this paper is to study how to

The work of Z. Ding was supported by a Marie Curie Outgoing International Fellowship within the 7th European Community Framework Programme and the UK EPSRC under grant number EP/I037423/1. The work of I. Esnaola, S. Perlaza and H. V. Poor was supported by the U. S. Army Research Office under MURI Grant W911NF-11-1-0036, the U. S. Air Force Office of Scientific Research under MURI Grant FA9550-09-1-0643, the U. S. National Science Foundation under Grant DMS-1118605, and the U. S. Office of Naval Research under Grant N00014-12-1-0767. efficiently distribute such power among the multiple users and investigate the impact of these power allocation strategies on the system performance.

We first develop a non-cooperative individual transmission strategy, in which the relaying transmission to the $i$-th destination is powered by using only the energy harvested from the $i$-th source. Such a simple power allocation scheme will serve as a benchmark for other more sophisticated strategies developed in the paper. The decode-and-forward (DF) protocol is considered, and an exact expression for the outage probability achieved by such a scheme is obtained. Then, the performance of an equal power allocation scheme is investigated, in which the relay distributes the accumulated power harvested from the sources evenly among relaying transmissions. The advantage of such a scheme is that a user pair with poor channel conditions can be helped since more relay transmission power will be allocated to them compared to the individual transmission strategy. Exact expressions for the outage performance achieved by this transmission scheme are obtained. Analytical results show that the equal power allocation scheme always outperforms the individual transmission strategy.

Compared to the equal power allocation scheme, a more efficient strategy is that in which the relay serves a user with a better channel condition first, and helps a user with a worse channel condition afterwards if there is any power left at the relay. This sequential water filling scheme can achieve the optimal outage performance for the user with the best channel conditions, and also maximize the number of successful destinations. Surprisingly it can also be proved that such a scheme minimizes the worst user outage probability. Several bounds are developed for the average outage probability achieved by such a scheme, and the developed analytical results show that this scheme can achieve the best performance, when global channel stage information is available.

\section{ENERGY HARVESTING RELAYING TRANSMISSIONS}

Consider an energy harvesting communication scenario with $M$ source-destination pairs and one relay. Each node is equipped with a single antenna. Each source communicates with its destination via the relay using orthogonal channels, such as different time slots. All channels are assumed to be quasi-static Rayleigh fading, and large scale path loss will be considered only in Section IV in order to simplify the analytical development.

The basic idea of energy harvesting relaying is that an energy constrained relay recharges its battery by using the 
energy from its observations. Among the various energy harvesting relaying models, we focus on power splitting [6], [7]. Specifically, the cooperative transmission consists of two time slots of duration $\frac{T}{2}$. At the end of the first phase, the relay splits the observations from the $i$-th transmitter into two streams, one for energy harvesting and the other for detection. Let $\theta_{i}$ denote the power splitting coefficient for the $i$-th user pair, i.e. $\theta_{i}$ is the fraction of observations used for energy harvesting. At the end of the first phase, the relay's detection is based on the following observation:

$$
y_{r, i}=\sqrt{\left(1-\theta_{i}\right) P_{i}} h_{i} s_{i}+n_{r, i},
$$

where $P_{i}$ denotes the transmission power at the $i$-th source, $h_{i}$ denotes the channel gain between the $i$-th source and the relay, $s_{i}$ is the source message with unit power, and $n_{r, i}$ denotes additive white Gaussian noise (AWGN) with unit variance. As discussed in [7], such noise consists of the baseband AWGN as well as the sampled AWGN due to the radio-frequency band to baseband signal conversion. We consider a pessimistic case in which power splitting reduces only the signal power, but not the noise power, which can provide a lower bound for relaying networks in practice.

The data rate at which the relay can decode the $i$-th source's signal is

$$
R_{r, i}=\frac{1}{2} \log \left(1+\left(1-\theta_{i}\right) P_{i}\left|h_{i}\right|^{2}\right),
$$

and the parameter $\theta_{i}$ can be set to satisfy the criterion $R_{r, i}=$ $R$, i.e.,

$$
\theta_{i} \triangleq 1-\frac{2^{2 R}-1}{P_{i}\left|h_{i}\right|^{2}},
$$

where $R$ is the targeted data rate. The reason for the above choice of $\theta_{i}$ can be justified as follows. A larger value of $\theta_{i}$ yields more energy reserved for the second phase transmissions, and therefore is beneficial to improve the performance at the destination. On the other hand, a larger value of $\theta_{i}$ reduces the signal power for relay detection and hence degrades the receptional reliability at the relay. For that reason, a reasonable choice is to use a $\theta_{i}$ that assumes successful detection at the relay, i.e., $R_{r, i} \geq R$.

At the end of the first phase, the relay harvests the following amount of energy from the $i$-th source:

$$
E_{H, i}=\eta P_{i}\left|h_{i}\right|^{2} \theta_{i} \frac{T}{2},
$$

where $\eta$ denotes the energy harvesting efficiency factor. During the second time slot, this energy can be used to power the relay transmissions. However, how to best use such harvested energy is not a trivial problem, since different strategies will have different impacts on the system performance. In the following subsection, we first introduce a non-cooperative individual transmission strategy, which serves as a benchmark for the transmission schemes proposed later.

\section{A non-cooperative individual transmission strategy}

One straightforward strategy to use the harvested energy is allocating the energy harvested from the $i$-th source to the relaying transmission to the $i$-th destination, i.e., the relaying transmission power for the $i$-th destination is

$$
P_{r i} \triangleq \frac{E_{H, i}}{\frac{T}{2}}=\eta P_{i}\left|h_{i}\right|^{2} \theta_{i} .
$$

During the second time slot, the DF relay forwards the $i$ th source message if the message is reliably detected at the relay, i.e. $\left|h_{i}\right|^{2}>\epsilon$, where $\epsilon=\frac{2^{2 R}-1}{P_{i}}$. Therefore, given a successful detection at the relay, the $i$-th destination receives the observation, $\sqrt{P_{r i}} g_{i} s_{i}+n_{d, i}$, which yields a data rate at the $i$-th destination of

$$
R_{d, i}=\frac{1}{2} \log \left(1+P_{r i}\left|g_{i}\right|^{2}\right)
$$

where $g_{i}$ denotes the channel between the relay and the $i$ th destination and $n_{d, i}$ denotes the noise at the destination. For notational simplicity, it is assumed that the noise at the destination has the same variance as that at the relay. The outage probability for the $i$-th user pair can be expressed as

$$
\begin{aligned}
\mathcal{P}_{i, I}=\operatorname{Pr} & \left(\frac{1}{2} \log \left(1+P_{i}\left|h_{i}\right|^{2}\right)<R\right)+\operatorname{Pr}\left(\frac{1}{2} \log (1\right. \\
& \left.\left.+P_{i}\left|h_{i}\right|^{2}\right)>R, \frac{1}{2} \log \left(1+P_{r i}\left|g_{i}\right|^{2}\right)<R\right) .
\end{aligned}
$$

The following proposition characterizes the outage of such a strategy.

Proposition 1: The use of the non-cooperative individual transmission strategy yields an outage probability at the $i$-th destination of

$$
\mathcal{P}_{i, I}=1-\frac{e^{-\frac{a}{P_{i}}}}{P_{i}} \sqrt{\frac{4 a P_{i}}{\eta}} \mathbf{K}_{1}\left(\sqrt{\frac{4 a}{\eta P_{i}}}\right),
$$

where $a=2^{2 R}-1$ and $\mathbf{K}_{n}(\cdot)$ denotes the modified Bessel function of the second kind with order $n$. The worst and best outage performance among the $M$ users are $1-\left(1-\mathcal{P}_{i, I}\right)^{M}$ and $\left(\mathcal{P}_{i, I}\right)^{M}$, respectively.

Proof: Please refer to [8].

With some algebraic manipulations, the averaged outage performance achieved by the non-cooperative individual strategy can be approximated at high SNR as

$$
\begin{aligned}
\mathcal{P}_{i, I} & \approx 1-(1-\epsilon)\left(1+\frac{2 \epsilon}{\eta} \ln \sqrt{\frac{\epsilon}{\eta}}\right) \\
& \approx \epsilon\left(1-\frac{2}{\eta} \ln \sqrt{\frac{\epsilon}{\eta}}\right) .
\end{aligned}
$$

Note that $\epsilon$ is proportional to $\frac{1}{S N R}$, which means that the averaged outage probability for the individual transmission scheme decays as $\frac{\log S N R}{S N R}$. Strategies achieving better outage performance will be introduced in the following sections.

\section{CENTRAlized MECHANISMS FOR POWER ALlOCATION}

Recall that each user uses the power splitting fraction $\theta_{i}=$ $1-\frac{2^{2 R}-1}{P_{i}\left|h_{i}\right|^{2}}$, which implies that the total power reserved at the relay at the end of the first phase is

$$
P_{r}=\sum_{i=1}^{N} \frac{E_{H, i}}{\frac{T}{2}}=\sum_{i=1}^{N} \eta P_{s}\left|h_{i}\right|^{2} \theta_{i}
$$


where $N$ denotes the number of sources whose information can be reliably detected at the relay. Note that $N$ is a random variable whose value depends on the instantaneous source-relay channel realizations. To simplify the analysis, it is assumed that all the source transmission powers are the same $P_{i}=P_{s}$. In the following, we study how to distribute such power among the users based on various criteria. Specifically, an equal power allocation strategy is introduced first, and then we will investigate the water filling based strategy which achieves a better outage performance but requires high complexity.

\section{A. Equal power allocation}

In this strategy, the relay allocates the same amount of power to each user, i.e., $P_{r i}=\frac{1}{N} \sum_{i=1}^{N} \eta P_{s}\left|h_{i}\right|^{2} \theta_{i}$. The advantage of such a strategy is that there is no need for the relay to know the relay-destination channel information, which can reduce the system overhead significantly, particularly in a multi-user system. The following theorem describes the outage performance achieved by such a power allocation scheme.

Theorem 1: Based on the equal power allocation, the outage probability for the $i$-th destination is given by

$$
\begin{aligned}
\mathcal{P}_{i, I I}= & \sum_{n=1}^{M} \frac{1}{(n-1) !}\left((n-1) !-2\left(\frac{b_{n}}{P}\right)^{\frac{n}{2}} \mathbf{K}_{n}\left(2 \sqrt{\frac{b_{n}}{P}}\right)\right) \\
& \times \frac{(M-1) !}{(n-1) !(M-n) !} e^{-n \epsilon}\left(1-e^{-\epsilon}\right)^{M-n}+\left(1-e^{-\epsilon}\right),
\end{aligned}
$$

where $b_{n}=\frac{n a}{\eta}$.

Proof: Please refer to [8].

With some algebraic manipulations, the high SNR approximation of $\mathcal{P}_{i, I I}$ can be obtained as follows

$$
\mathcal{P}_{i, I I} \approx\left(1+\frac{M}{(M-1) \eta}\right) \epsilon,
$$

which means that the equal power allocation scheme can achieve better performance than the non-cooperative scheme, i.e. a faster rate of decay, $\frac{1}{S N R}$, since $\epsilon$ is proportional to SNR.

Based on Theorem 1, we also obtain the best outage and worst outage performance among the $M$ users achieved by the equal power allocation scheme as follows.

Proposition 2: Based on the use of the equal power allocation, the outage probability of the user with the best channel conditions among the $M$ users is

$$
\mathcal{P}_{\text {best }, I I}=\sum_{n=1}^{M} \frac{2}{(n-1) !} \sum_{i=0}^{n}\left(\begin{array}{c}
n \\
i
\end{array}\right)(-1)^{i}\left(\frac{i b_{n}}{P}\right)^{\frac{n}{2}}
$$

$\times \mathbf{K}_{n}\left(2 \sqrt{\frac{i b_{n}}{P}}\right) \frac{M ! e^{-n \epsilon}}{n !(M-n) !}\left(1-e^{-\epsilon}\right)^{M-n}+\left(1-e^{-\epsilon}\right)^{M}$,

and the worst outage performance among the $M$ users is

$$
\begin{aligned}
\mathcal{P}_{\text {worst }, I I} & =\frac{1}{(M-1) !}\left((M-1) !-2\left(\frac{M b_{M}}{P}\right)^{\frac{M}{2}}\right. \\
& \left.\times \mathbf{K}_{M}\left(2 \sqrt{\frac{M b_{M}}{P}}\right)\right) e^{-M \epsilon}+1-e^{-M \epsilon} .
\end{aligned}
$$

Proof: Please refer to [8].

\section{B. Sequential water filling based power allocation strategy}

Provided that the relay has access to global channel state information, a more efficient strategy that maximizes the number of successful destinations can be designed as follows. First recall that in order to ensure the successful detection at the $i$-th destination, the relay needs to allocate the relaying transmission power $P_{i, \text { targeted }}=\frac{2^{2 R}-1}{\left|g_{i}\right|^{2}}$ to the $i$-th destination. Suppose that $n$ sources can deliver their information to the relay reliably, and the required relaying transmission power for these $n$ destinations can be ordered as

$$
\frac{2^{2 R}-1}{\left|g_{(1)}\right|^{2}} \geq \cdots \geq \frac{2^{2 R}-1}{\left|g_{(n)}\right|^{2}} .
$$

The sequential water filling power allocation strategy is described in the following. The relay first serves the destination with the strongest channel by allocating power $\frac{2^{2 R}-1}{\left|g_{(n)}\right|^{2}}$ to it, if the total harvested energy at the relay is larger than or equal to $\frac{2^{2 R}-1}{\left|g_{(n)}\right|^{2}}$. And then the relay tries to serve the destination with the second strongest channel with the power $\frac{2^{2 R}-1}{\left|g_{(n-1)}\right|^{2}}$, if possible. Such a power allocation strategy continues until either all users are served or there is not enough power left at the relay. If there is any power left, such energy is reserved at the relay, where it is assumed that the capacity of the relay battery is infinite.

The probability of having $m$ successful receivers among $n$ users can be expressed as

$\operatorname{Pr}\left(\sum_{i=1}^{m} \frac{2^{2 R}-1}{\mid g_{\left.(n-i+1)\right|^{2}}}<P_{r}, \sum_{i=1}^{m+1} \frac{2^{2 R}-1}{\left|g_{(n-i+1)}\right|^{2}}>P_{r}, N=n\right)$,

from which the averaged number of successful destinations can be calculated by carrying out the summation among all possible choices of $m$ and $n$. Evaluating the above expression is quite challenging, mainly because of the complexity of the density function of the sum of inverse exponential variables. However, explicit analytical results for such a power allocation scheme can be obtained based on other criteria. Particularly we are interested in the outage performance achieved by the water filling strategy.

Although such a water filling power allocation scheme is designed to maximize the number of successful destinations, it can also minimize the outage probability for the user with the best channel conditions, since such a user is the first to be served and has access to the maximal relaying power. The following proposition provides an explicit expression for such a outage probability.

Proposition 3: With the sequential water filling power allocation strategy, the outage probability for the user with the best channel conditions is

$$
\begin{aligned}
\mathcal{P}_{\text {best }, I I I}= & \sum_{n=1}^{M} \frac{2}{(n-1) !} \sum_{i=1}^{n}\left(\begin{array}{c}
n \\
i
\end{array}\right)(-1)^{i}\left(\frac{i \tilde{b}}{P}\right)^{\frac{n}{2}} \mathbf{K}_{n}\left(2 \sqrt{\frac{i \tilde{b}}{P}}\right) \\
& \times \frac{M !}{n !(M-n) !} e^{-n \epsilon}\left(1-e^{-\epsilon}\right)^{M-n}+\left(1-e^{-\epsilon}\right)^{M}
\end{aligned}
$$

where $\tilde{b}=\frac{a}{\eta}$.

Proof: Please refer to [8]. 
The optimality of the water filling scheme in terms of the number of successful destinations and the performance for the user with the best channel conditions is straightforward to demonstrate. However, it is surprising that the performance of the water filling scheme for the user with the worst outage probability is the same as that attained for the worst user with the optimal strategy, as shown in the following lemma.

Lemma 1: Denote by $\mathcal{P}_{i}(\mathrm{~s})$ the outage probability for the $i$-th user achieved by a power allocation strategy $\mathrm{s}$, where $\mathrm{s} \in$ $\mathcal{S}$ and $\mathcal{S}$ contains all possible strategies. Define $\mathcal{P}_{\text {worst }}(\mathrm{s}) \triangleq$ $\max \left\{\mathcal{P}_{\mathrm{i}}(\mathrm{s}), \mathrm{i} \in\{1, \cdots, \mathrm{M}\}\right\}$ and $\mathcal{P}_{\text {worst,III }}$ as the worst user performance achieved by the sequential water filling scheme. Then $\mathcal{P}_{\text {worst }, I I I}=\min \left\{\mathcal{P}_{\text {worst }}(\mathrm{s}), \mathrm{s} \in \mathcal{S}\right\}$.

Proof: Please refer to [8].

The following proposition provides upper and lower bounds of the outage performance of the users with the worst channel conditions.

Proposition 4: The outage probability for the user with the worst channel conditions achieved by the water filling strategy can be upper bounded by

$$
\begin{array}{r}
\mathcal{P}_{\text {worst }, I I I}<e^{-M \epsilon} \int_{0}^{\infty}\left(1-e^{-\frac{M^{2}}{w}}\right. \\
\left.-M \int_{\frac{w}{M}}^{w} \frac{e^{-\frac{(M-1)^{2}}{w-v}-\frac{1}{v}}}{v^{2}} d v\right) f_{w}(w) d w+1-e^{-M \epsilon},
\end{array}
$$

and lower bounded by

$$
\begin{array}{r}
\mathcal{P}_{\text {worst }, I I I}>\left(1-\frac{2}{(M-1) !}\left(\frac{M \epsilon}{\eta}\right)^{\frac{M}{2}}\right. \\
\left.\mathbf{K}_{M}\left(2 \sqrt{\frac{M \epsilon}{\eta}}\right)\right) \\
\times e^{-M \epsilon}+1-e^{-M \epsilon},
\end{array}
$$

where $f_{w}(w)=\frac{1}{(M-1) !}\left(\frac{\epsilon}{\eta}\right)^{M} w^{M-1} e^{-\frac{\epsilon}{\eta} w}$.

Proof: Please refer to [8].

While the expression in (12) can be evaluated by numerical methods, it is difficult to carry out asymptotic studies for such an expression with integrals, and the following proposition provides a bound slightly looser than (12) that enables asymptotic analysis.

Proposition 5: The outage probability for the user with the worst channel conditions achieved by the water filling strategy can be upper bounded as follows:

$$
\begin{gathered}
\mathcal{P}_{\text {worst }, \text { III }}<\left(1-\frac{2}{(M-1) !}\left(\frac{M^{2} \epsilon}{\eta}\right)^{\frac{M}{2}}\right. \\
\times \mathbf{K}_{M}\left(2 \sqrt{\frac{M^{2} \epsilon}{\eta}}\right)-\frac{M}{(M-2) !}\left(\frac{\epsilon}{\eta}\right)\left(\frac{M^{2} \epsilon}{\eta}\right)^{\frac{M-1}{2}} \\
\left.\times \mathbf{K}_{M-1}\left(2 \sqrt{\frac{M^{2} \epsilon}{\eta}}\right)\right) e^{-M \epsilon}+1-e^{-M \epsilon} .
\end{gathered}
$$

Proof: Please refer to [8].

It can be straightforward to verify that the upper and lower bounds converge at high SNR. For example, at high SNR, the upper bound which can be rewritten as

$$
\mathcal{P}_{\text {worst }, I I I}<\epsilon M\left(1+\frac{(M+1)}{2 \eta(M-1)}\right),
$$

which decays as $\frac{1}{S N R}$. On the other hand, the lower bound can be approximated as follows:

$$
\mathcal{P}_{\text {worst }, \text { III }}>\epsilon M\left(1+\frac{1}{\eta(M-1)}\right) .
$$

So the worst case outage probability achieved by the water filling scheme also decays as $\frac{1}{S N R}$.

\section{NumeriCAL RESUlts}

In this section, computer simulations that illustrate the performance of those energy harvesting relaying protocols described in the previous sections are described.

We first study the accuracy of the developed analytical results. Specifically in Fig. 1, the outage performance achieved by the individual transmission scheme and the equal power allocation scheme is shown as a function of SNR. All the channel coefficients are assumed to be complex Gaussian with zero means and unit variances. The targeted data rate is $R=2$ bits per channel use (BPCU), and the energy harvesting efficiency is set as $\eta=1$. As can be seen from the figures the developed analytical results exactly match the simulation results, which demonstrates the accuracy of the developed analytical results.

Comparing the two cases in Fig. 1, we find that the use of the equal power allocation strategy improves the outage performance. Consider the averaged outage performance as an example. When the SNR is $40 \mathrm{~dB}$, the use of the individual transmission scheme realizes outage probability of $1 \times 10^{-2}$,

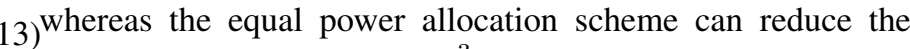
outage probability to $3 \times 10^{-3}$. Such a phenomenon confirms the asymptotic results shown in Section IV.A. Specifically the outage probability achieved by the individual transmission scheme decays with the SNR at a rate $\frac{1}{S N R}(1+2 \ln S N R)$, but the equal power allocation scheme achieves a faster rate of decay, $\frac{1}{S N R}$.

When more source-destination pairs join in the transmission, it is more likely to have some nodes with extreme channel conditions, which explains the phenomenon in Fig. 1 that with a larger number of user pairs, the best outage performance improves but the worst outage performance degrades. The impact of the number of user pairs on the average outage performance can also be observed in the figure. For the individual transmission scheme, there is no cooperation among users, so the number of user pairs has no impact on the average outage performance. On the other hand, it is surprising to find that an increase in the number of users yields only a slight improvement in the performance of the equal power allocation scheme, which might be due to the fact that power allocation can improve the transmission from the relay to the destinations, but not the source-relay transmissions.

In Fig. 2, the performance of the water filling scheme is studied. The same simulation setup as in the previous figures is used. Firstly the upper and lower bounds developed in Lemma 4 are compared to the simulation results in Fig. 2.a. As can be seen from the figure, the lower bound developed in (13) and the upper bound in (12) are very tight, whereas the upper bound in (14) is reasonably close to the actual performance. Recall that the reason that the bounds in (12) and (13) are 


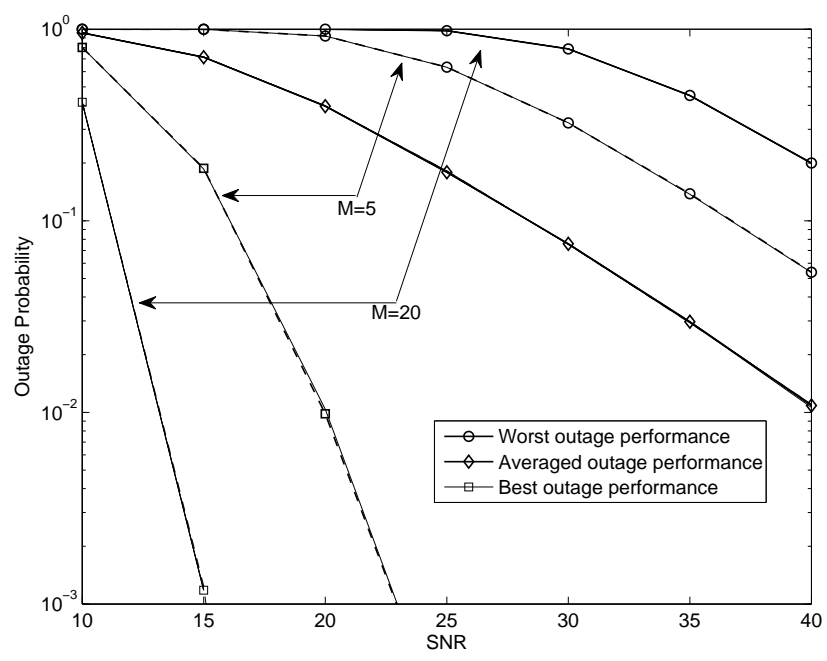

(a) Individual transmission

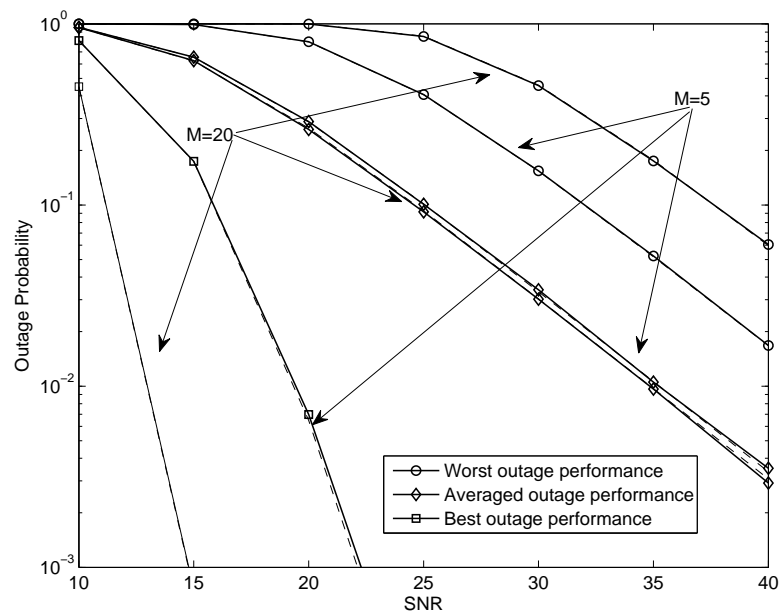

(b) Equal power allocation

Fig. 1. Outage probabilities achieved by the individual transmission scheme and the equal power allocation scheme. $R=2 \mathrm{BPCU}$. The solid curves are for the simulation results and the dashed ones are for the analytical results.

tight is because the sum of inverse exponential variables is an unbounded variable. On the other hand, the gap between the upper bounds in (14) and (12) is due to the applied approximation. In Fig. 2.b, the outage performance based on different criteria is shown for the water filling scheme. Comparing Figures 1 and 2, we can see that the use of the water filling scheme yields the best performance.

\section{CONClusion}

In this paper, we have considered several power allocation strategies for a cooperative network in which multiple sourcedestination pairs communicate with each other via an energy harvesting relay. The non-cooperative individual transmission scheme results in a outage performance decaying as $\frac{\log S N R}{S N R}$, whereas the centralized power allocation strategies ensure that the outage probability decays at a faster rate $\frac{1}{S N R}$, and the water filling scheme can achieve optimal performance in terms of a few criteria.

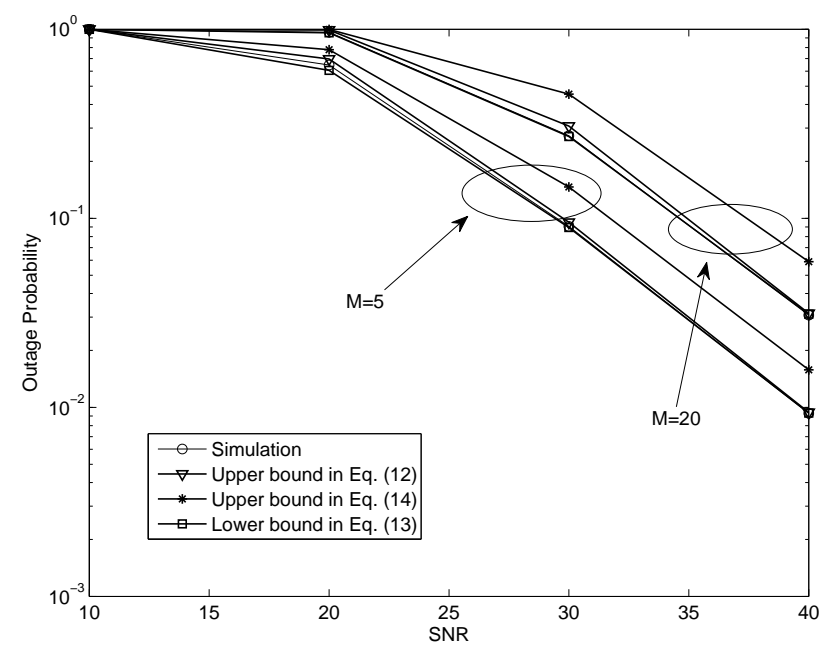

(a) Bounds for worst outage performance

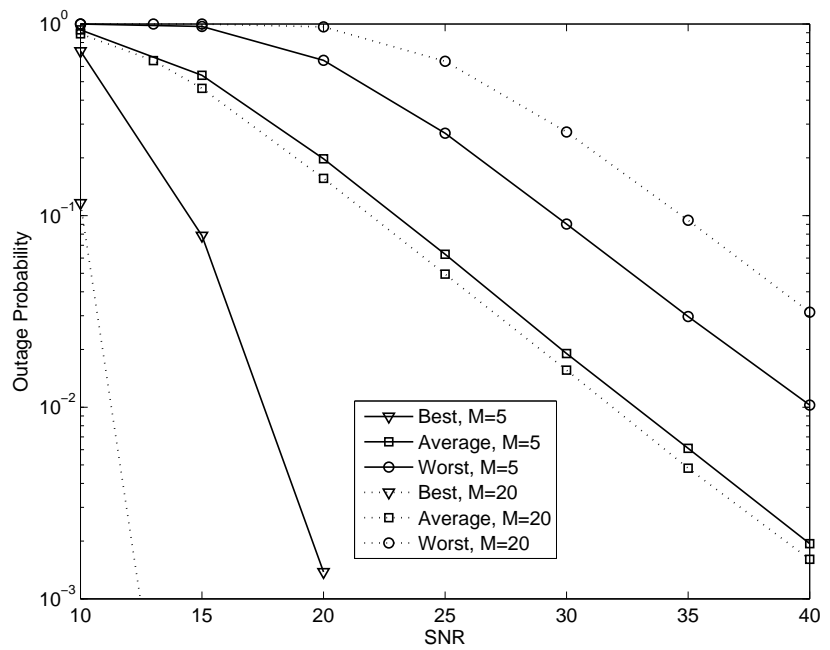

(b) Outage performance

Fig. 2. Outage probability achieved by the water filling transmission scheme. $R=2$ BPCU.

\section{REFERENCES}

[1] L. R. Varshney, "Transporting information and energy simultaneously," in Proc. IEEE Int. Symp. Inf. Theory (ISIT), Toronto, Canada, Jul. 2008.

[2] P. Grover and A. Sahai, "Shannon meets Tesla: wireless information and power transfer," in Proc. IEEE Int. Symp. Inf. Theory (ISIT), Austin, TX, Jun. 2010.

[3] L. Liu, R. Zhang, and K.-C. Chua, "Wireless information transfer with opportunistic energy harvesting," IEEE Trans. Wireless Commun., vol. 12, no. 1, pp. $288-300$, Jan. 2013.

[4] R. Zhang and C. K. Ho, "MIMO broadcasting for simultaneous wireless information and power transfer," in Proc. IEEE Globecom, Houston, TX, Dec. 2011.

[5] Z. Xiang and M. Tao, "Robust beamforming for wireless information and power transmission," IEEE Wireless Commun. Letters, vol. 1, no. 4, pp. 372-375, Jan. 2012.

[6] X. Zhou, R. Zhang, and C. K. Ho, "Wireless information and power transfer: Architecture design and rate-energy tradeoff," [online]. Available: http://arxiv.org/abs/1205.0618, 2012.

[7] A. A. Nasir, X. Zhou, S. Durrani, and R. A. Kennedy, "Relaying protocols for wireless energy harvesting and information processing," [online]. Available: http://arxiv.org/abs/1212.5406, 2012.

[8] Z. Ding, S. M. Perlaza, I. Esnaola, and H. V. Poor, "Power allocation strategies in energy harvesting wireless cooperative networks," IEEE Trans. Wireless Commun., (submitted). 